D. BagANOFF

M. NATHENSON

\title{
CONSTITUTIVE RELATIONS FOR STRESS AND HEAT FLUX IN A SHOCK WAVE
}

\section{CASE FILE COPY}

Supported by the

IUNE National Aeronautics and Space Administration 
Department of Aeronautics and Astronautics

Stanford University

Stanford, California

CONSTITUTIVE RELATIONS FOR STRESS AND

HEAT FLUX IN A SHOCK WAVE

by

D. Baganoff

and

M. Nathenson

SUDAAR NO. 379

June 1969

This research was sponsored by the National Aeronautics and Space Administration under Research Grant NGR 05-020-245. 


\begin{abstract}
A form of Maxwell's moment method is used to obtain constitutive relations for stress and heat flux for a gas passing through a shock wave. The $c_{x}^{2}$ moment of the Boltzmann equation together with several physical approximations are used to obtain a nonlinear expression relating stress and deformation rate, with Mach number as a parameter. It is shown that the constitutive relations for both stress and heat flux reduce to the Navier-Stokes relations for the case $\mathrm{M}=1$ and $|\tau / \mathrm{p}| \ll 1$, and differ from these relations by as much as a factor of two for $|\tau / p|=1$. Theoretical density profiles for shock-wave Mach numbers between 2.8 and 8 are compared with experimental data from shock waves in argon and the agreement is shown to be excellent, thus supporting the use of the proposed constitutive relations. The physical significance of the approximations used and areas for further improvement in the theory are discussed.
\end{abstract}




\section{CONTENTS}

CHA PTER

PAGE

I. INTRODUCTION . . . . . . . . . . . . . . 1

II. MOMENT EQUATIONS . . . . . . . . . . . . . 4

A. Expression for $\left\langle\mathrm{c}_{\mathrm{x}}^{3}\right\rangle$. . . . . . . . . . . . . 8

B. Approximation for $\left\langle\mathrm{v}_{\mathrm{x}}^{3}\right\rangle$. . . . . . . . . . 10

III. SHOCK-WAVE STRUCTURE, $Q=5 / 6 . . . . . . . . .18$

IV. EXTENSION, $\mathrm{Q}=\mathrm{Q}(\mathrm{M})$. . . . . . . . . . . 23

V. DISCUSSION ....... . . . . . . . . 30

REFERENCES ................. . . 33 


\section{INTRODUCTION}

An important step in the application of the moment method employed by Mott-Smith to analyze shock structure, and which was later extended by Lees to treat problems in rarefied gas dynamics, is the selection of an approximate functional form for the distribution function ${ }^{1,2}$. The functional form selected must satisfy physical requirements imposed by boundaries and possess sufficient flexibility, in the form of free parameters, to satisfy a desired number of moments of the Boltzmann equation. The power of the method resides in the fact that for situations involving extreme translational nonequilibrium one can often construct a distribution function, relying mainly on intuition and a physical understanding of the problem, which is a better approximation to the actual flow than one obtained through a more rational approach such as the Chapman-Enskog procedure. Mott-Smith's bimodal distribution and Lees' two-sided Maxwellian are wellknown examples of distribution functions constructed on this basis.

A useful interpretation for explaining the effectiveness of this particular approach to Maxwell's moment method is that it provides, in effect, a mechanism for closing a set of moments of the Boltzmann equation by expressing the unknown higher order moments in terms of the "known" lower order moment through the assumed form of the distribution function. For example, the moment $\left\langle c_{x}^{3}\right\rangle$ (or $\left\langle c_{x}^{4}\right\rangle$ ) is the unknown moment in the Mott-Smith solution to the shockstructure problem and the imodal distribution allows one, in principle, to express $\left\langle c_{x}^{3}\right\rangle$ in terms of the lower order moments, i. e., gas dynamic variables. 
Since the specific information needed to close a set of moments of the Boltzmann equation is one or more particular higher order moments of the distribution function, this raises the following question: Is it easier to guess the moment needed, e.g. $\left\langle\mathrm{c}_{\mathrm{x}}^{3}\right\rangle$, or to guess the distribution $f$ and then compute the moment needed? For problems such as Couette flow or heat conduction in a rarefied gas, the latter is probably most likely true since solid surfaces play such important roles, and it seems natural that processes occurring at a surface should enter directly into the assumed form for f. For the shock-structure problem, there is reason to believe the former is true because of the absence of solid boundaries and because of the fact that one of the unknown moments $\left\langle c_{x}^{3}\right\rangle$ is directly related to physical quantities in the flow; and, as will be seen below, one can therefore estimate this moment rather well without having to select an approximate $\mathbf{f}$.

The aim of this paper is therefore to present an analysis of the shockstructure problem with a basically different emphasis on the Maxwell moment method than that employed by Mott-Smith. In fact, the aim is to show that for steady, one-dimensional flow one can close the set of moments of the Boltzmann equation, at the "Mott-Smith" level of approximation, by approximating the unknown moments directly rather than approximating f. The primary reason for emphasizing this approach is that one arrives at new constitutive laws for stress and heat flux, which replace the Navier-Stokes relations, and consequently all of the advantages that accrue from the continuum description of a gas are retained. In addition, it becomes possible to see directly the physical significance 
of the solution and specifically where the analysis could be improved, in contrast to previous solutions where the outcome of the analysis is simply a shockwave profile for specific assumptions. 


\section{MOMENT EQUATIONS}

For a steady, one-dimensional situation, the "first" five moments of the Boltzmann equation are given by

$$
\begin{aligned}
& \frac{d}{d x}(\rho u)=0 \\
& \frac{d}{d x}\left(p+\rho u^{2}-\tau\right)=0 \\
& \frac{d}{d x}\left[\rho u\left(h+\frac{1}{2} u^{2}\right)+(q-\tau u)\right]=0 \\
& \frac{d}{d x} p<c_{x}^{3}>m \Delta\left[c_{x}^{2}\right] \\
& \frac{d}{d x} \rho<c_{x}^{2} c^{2}>=m \Delta\left[c_{x} c^{2}\right],
\end{aligned}
$$

where $p, \rho, h, u, \tau$ and $q$ are the standard gas dynamic variables, $m$ is the mass of the molecule, $\Delta\left[c_{x}^{2}\right]$ represents the $c_{x}^{2}$ moment of the collision integral, and $c_{x}$ is the molecular speed in the $x$-direction (the direction of flow). The terms in Eqs. (1d) and (1e) arising from the collision integral can be expressed in terms of $\tau$ and $q$ for Maxwell molecules ${ }^{3}$, i.e.,

$$
\begin{aligned}
& m \Delta\left[c_{x}^{2}\right]=\frac{p}{\mu} \\
& m \Delta\left[c_{x} c^{2}\right]=\frac{2 p}{\mu}\left(\tau u-\frac{2}{3} q\right),
\end{aligned}
$$

where the constants describing the intermolecular potential have been replaced by the coefficient of viscosity $\mu$, as defined by the Chapman-Enskog procedure. 
This simplification is frequently used in the approximate theories of rarefied gas dynamics.

In order to show where the present work could be generalized in the discussion below, by removing the restriction to Maxwell molecules, it is convenient to define here the quantity $\tau^{*}$ by the relation

$$
\mathrm{m} \Delta\left[\mathrm{c}_{\mathrm{x}}^{2}\right]=\frac{\mathrm{p}}{\mu} \tau^{*}
$$

Use of Eq. (3) will therefore preserve the algebraic form obtained for Maxwell molecules since $\tau^{*}=\tau$ in that case. In general, one would have to obtain the relation between $\tau^{*}$ and $\tau$ (and possibly other variables) for a particular intermolecular potential.

Equations (1) and (2) together with the relations for a thermally and calorically perfect gas

$$
\begin{aligned}
& \mathrm{p}=\rho \mathrm{RT} \\
& \mathrm{h}=\mathrm{c}_{\mathrm{p}} \mathrm{T}
\end{aligned}
$$

do not form a complete set of equations since there are more unknowns (9) than equations (7); that is, the moments $\left\langle c_{x}^{3}\right\rangle$ and $\left\langle c_{x}^{2} c^{2}\right\rangle$ are the extra unknowns. If the approach used by Mott-Smith were pursued at this point, then the extra unknowns would be evaluated from an assumed distribution function. Rather than follow this approach, let us first consider the information contained in Eqs. (1d) and (1e).

Using the relations for Maxwell molecules (2a) and (2b), we have from (1d) and (1e) 


$$
\begin{aligned}
& \frac{p}{\mu}=\frac{d}{d x} p<c_{x}^{3}> \\
& \frac{2 p}{\mu}\left(\tau u-\frac{2}{3} q\right)=\frac{d}{d x} p<c_{x}^{2} c^{2}>0
\end{aligned}
$$

Since $\tau$ is determined by (5a) therefore $q$ is essentially determined by (5b). If the moments $\left\langle\mathrm{c}_{\mathrm{x}}^{3}\right\rangle$ and $\left\langle\mathrm{c}_{\mathrm{x}}^{2} \mathrm{c}^{2}\right\rangle$ could somehow be expressed as functions of the gas dynamic variables, it is clear that Eqs. (5) would yield constitutive laws for stress and heat flux. It is therefore desirable to preserve the identities of (5a) and $(5 \mathrm{~b})$ during all algebraic manipulations in order to identify the resulting constitutive relations, and in addition, to remind one that all approximations introduced will affect only these relations. Once these relations are established, it is a simple procedure to compute shock-wave profiles with the complete set of equations and thereby check the approximations used by comparing the results with experimental data.

Since Eq. (5b) is an equation for the heat flux q, it would seem that a far better approximation for $q$ than that obtained by guessing $f$ and computing $\left\langle\mathrm{c}_{\mathrm{x}}^{2} \mathrm{c}^{2}\right\rangle$ would be to assume the total enthalpy is conserved within a shock-wave profile and use the energy equation (1c) to obtain

$$
q=\pi u
$$

Equation (6) has been shown by Liepmann, Narasimha, and Chahine ${ }^{4}$ to be an excellent approximation for the Navier-Stokes equations, and Sherman and Talbot 5 have shown the approximation is very good for several continuum theories as well as for the Mott-Smith solution. Here we will assume Eq. (6) holds in general and rely on the comparisons given below to justify this selection. 
Using the energy equation consistent with (6) and the notation defined by (3), the set of equations given by (1) becomes

$$
\begin{aligned}
& \frac{d}{d x}(\rho u)=0 \\
& \frac{d}{d x}\left(p+\rho u^{2}-\tau\right)=0 \\
& \frac{d}{d x}\left(h+\frac{1}{2} u^{2}\right)=0 \\
& T^{*}=\frac{\mu}{p} \frac{d}{d x} p<c_{x^{3}}^{3} \\
& q=\tau u .
\end{aligned}
$$

Displayed in the above form, we see that the constitutive laws for stress and heat flux must arise from Eqs. (7d) and (7e) respectively. In fact, one should expect to recover the Navier-Stokes relations

$$
\begin{aligned}
& \tau^{o}=\frac{4}{3} \mu \frac{d u}{d x} \\
& q^{o}=-k \frac{d T}{d x}
\end{aligned}
$$

from these two equations as the strength of the shock wave approaches zero, i.e. , as the Mach number approaches unity. The nature of this limit process is not obvious when inspecting Eq. (7d); however, the expression can be cast in a form for which the limit process becomes evident immediately. This can be seen if we express $\left\langle\mathrm{c}_{\mathrm{x}}^{3}\right\rangle$ in terms of a central moment $\left\langle\mathrm{V}_{\mathrm{x}}^{3}\right\rangle$, where $\mathrm{V}_{\mathrm{x}}$ is the thermal speed given by 


$$
\mathrm{V}_{\mathrm{x}}=\mathrm{c}_{\mathrm{x}}-\mathrm{u}
$$

If we then let $\mathrm{M}_{\mathrm{s}} \rightarrow 1$ and $\mathrm{f} \rightarrow \mathrm{f}^{(0)}$, where $\mathrm{f}^{(0)}$ is the Maxwellian distribution, $\left\langle\mathrm{V}_{\mathrm{x}}^{3}\right\rangle$ becomes zero since $\mathrm{f}^{(0)}$ is an even function of $\mathrm{V}_{\mathrm{x}}$ and $\left\langle\mathrm{V}_{\mathrm{x}}^{3}\right\rangle$ is an odd moment. Therefore the remaining terms in Eq. (7d) must reduce exactly to (8a).

$$
\text { A. Expression for }\left\langle\mathrm{c}_{\mathrm{x}}^{3}\right\rangle
$$

The relation between $\left\langle\mathrm{c}_{\mathrm{x}}^{3}\right\rangle$ and $\left\langle\mathrm{V}_{\mathrm{x}}^{3}\right\rangle$ can be found directly from the definition (9), i.e. ,

$$
c_{x}^{3}=\left(V_{x}+u\right)^{3}
$$

and

$$
<c_{x}^{3}>=\left\langle V_{x}^{3}>+3 u<v_{x}^{2}>+u^{3}\right.
$$

since by definition $<\mathrm{V}_{\mathrm{x}}>=0$. Recalling that the $\mathrm{x}$-component of the pressure tensor is defined to be

$$
p_{x x}=\rho<V_{x}^{2}>p-\tau
$$

the above relation becomes

$$
\rho<c_{x}^{3}>=\rho<V_{x}^{3}>+3 u(p-\tau)+\rho u^{3}
$$

Equation (10) shows that $\left\langle\mathrm{c}_{\mathrm{x}}^{3}\right\rangle$ can be split into a sum of two parts, a central moment $\left\langle\mathrm{V}_{\mathrm{X}}^{3}\right\rangle$ and terms involving gas dynamic quantities.

Differentiating Eq. (10) with respect to $x$, expanding the right hand side, and using the continuity and momentum equations, (7a) and (7b), one obtains 


$$
\frac{d}{d x} \rho<c_{x}^{3}>=\frac{d}{d x} \rho<V_{x}^{3}>+\left[3(p-\tau)-\rho u^{2}\right] \frac{d u}{d x}
$$

Substituting this expression into (7d) and using the relation $\mathrm{a}^{2}=\frac{5}{3} \frac{\mathrm{p}}{\rho}$ for the isentropic speed of sound in a monatomic gas, we have

$$
T^{*}=\left[3(1-\tau / p)-\frac{5}{3} M^{2} \mu \frac{d u}{d x}+\frac{\mu}{p} \frac{d}{d x} p<V_{x}^{3}>\right.
$$

where $M$ is the local Mach number. The only difference between (7d) and (11) is that $\left\langle\mathrm{c}_{\mathrm{x}}^{3}\right\rangle$ has been replaced by the central moment $\left\langle\mathrm{V}_{\mathrm{x}}^{3}\right\rangle$, otherwise both equations are exact.

The limit value for $\tau^{*}$ for a weak shock wave can now be found from Eq. (11). When the shock Mach number is very close to one, the local Mach number $M$ is very nearly unity throughout the shock wave, $|\tau / p|$ is much less than one, and $\left\langle\mathrm{V}_{\mathrm{x}}^{3}\right\rangle$ is nearly zero. Therefore the right hand side of Eq. (11) reduces to $\frac{4}{3} \mu \frac{\mathrm{du}}{\mathrm{dx}}$, which is precisely the Navier-Stokes expression. For a general molecular force law, it is not clear that $\tau^{*}$ would also reduce to $\tau$ for $M_{S}=1$, but at least for Maxwell molecules it is certain that Eq. (11) yields the Navier-Stokes relation (8a).

This result immediately suggests that Eq. (11) could be quite useful for constructing an approximate expression for $T^{*}$ for larger Mach numbers. The assumption $\left\langle\mathrm{V}_{\mathrm{x}}^{3}\right\rangle=0$ gives a very good result for $\mathrm{M}_{\mathrm{s}}$ near one; thus any reasonable approximation to $\left\langle\mathrm{V}_{\mathrm{x}}^{3}\right\rangle$ should improve the expression, at least for a certain Mach number range. Here we can also see an advantage in using Eq. (11) over Eq. (7d) if we were to apply Mott-Smith's approach at this point by guessing a suitable f. That is, the entire expression for $\tau^{*}$ in Eq. (7d) 
would be approximate whereas only a part of the expression for $\tau^{*}$ in Eq. (11) would be approximate, and it would only be a minor contribution if $M_{s}$ is near one. In a certain sense one can view the correction as arising from a perturbation to the shock Mach number from unity.

A further inspection of Eq. (11) shows that it, unfortunately, is not well behaved for all $\mathrm{M}_{\mathrm{s}}$, since the term in square brackets becomes zero in the upstream part of the shock wave for $\mathrm{M}_{\mathrm{s}}=\sqrt{9 / 5}=1.34$. Therefore for strong shock waves the contribution to $T^{*}$ due to $\left\langle V_{x^{3}}^{3}\right.$ becomes very important to the total expression and it no longer can be considered as arising from a small perturbation.

It is of interest that the critical Mach number predicted by Eq. (11), when the term involving $\left\langle\mathrm{V}_{\mathrm{x}}^{3}\right\rangle$ is ignored, is close to the critical Mach numbers for the various continuum theories; e.g., Sherman and Talbot ${ }^{5}$ report that solutions of Grad's thirteen-moment equations are limited to the range $1 \leq \mathrm{M}_{\mathrm{s}} \lesssim 1.65$ and solutions to Burnett's equations are limited to the range $1 \leq \mathrm{M}_{\mathrm{S}} \lesssim 2.1$. In view of the fact that difficulty has been encountered with many schemes, for even moderate Mach numbers, it can be seen that an approximation for $\left\langle\mathrm{V}_{\mathbf{x}}^{3}\right\rangle$ must be chosen with care.

\section{B. Approximation for $\left\langle\mathrm{V}_{\mathrm{x}}^{3}\right\rangle$}

Before proceeding with approximate schemes for evaluating $\left\langle\mathrm{V}_{\mathrm{x}}^{3}\right\rangle$, the physical interpretation of $\rho\left\langle V_{x}^{3}\right\rangle$ should be stressed. Using the definition of the $\mathrm{x}$-component of the heat-flux vector, we have 


$$
q=\frac{1}{2} p<V_{x} V^{2}>
$$

Obviously for a one-dimensional flow, such as a shock wave, only the $\mathrm{x}$-component of the heat-flux vector is nonzero. However, this single component $q$ can be split into two parts, if we define $q^{\prime \prime}$ and $q^{\perp}$ by the relations

$$
\begin{aligned}
& q^{\prime \prime}=\frac{1}{2} p<V_{x}^{3}> \\
& q^{\perp}=\frac{1}{2} \rho<V_{x}\left(V_{y}^{2}+V_{z}^{2}\right)>.
\end{aligned}
$$

We can then speak of the parallel and perpendicular parts of the heat flux q; thus our problem requires an approximation for the parallel part of the heat flux as shown by (11) and (13a).

Since $q, q^{\prime \prime}$, and $q^{\perp}$ have identical dimensions, it is convenient to define the nondimensional quantity

$$
Q=q^{\prime \prime} / q,
$$

which exhibits the ratio of the parallel part to the total heat flux $q$. If $Q$ is assumed given, then $\left\langle V_{x}^{3}\right\rangle$ is obtained from Eqs, (13a) and (14), i. e. ,

$$
\rho<V_{x}^{3}>2 q Q
$$

The reason for introducing the ratio $Q$ becomes apparent once one evaluates the quantity for the Chapman-Enskog distribution $\mathrm{f}^{(1)}$. For this case we have

$$
\mathrm{Q}=3 / 5,
$$

i.e., $Q$ is strictly a constant everywhere in a flow field. The Maxwellian distribution $\mathrm{f}^{(0)}$ can be viewed as giving the "zeroth" approximation to $\left\langle\mathrm{V}_{\mathrm{x}}^{3}\right\rangle$ 
which is zero; and here $\mathrm{f}^{(1)}$ yields what can be considered to be the "first" approximation, i.e. ,

$$
\rho<V_{x}^{3}>=\frac{6}{5} q
$$

Since $q$ in a certain sense varies like $\frac{d T}{d x}$, it is easy to see that the second term in (11) is essentially $\frac{d^{2} T}{d x^{2}}$ while the first term is $\frac{d u}{d x^{3}}$ thus it makes sense that the second term should vanish first for weak shock waves as discussed above.

It is a straightforward procedure to substitute (17) into (11), use approximation (6), and obtain a new expression relating stress and deformation rate for a gas passing through a shock wave. The algebraic effort is reduced considerably if the following substitution is carried out first

$$
\frac{1}{\mathrm{p}} \frac{\mathrm{dq}}{\mathrm{dx}}=\frac{1}{\mathrm{p}} \frac{\mathrm{d}}{\mathrm{dx}} \mathrm{Tu}=\frac{\tau}{\mathrm{p}} \frac{\mathrm{du}}{\mathrm{dx}}+\frac{\mathrm{u}}{\mathrm{p}} \frac{\mathrm{d} \tau}{\mathrm{dx}}
$$

where $\frac{d \tau}{d x}$ is obtained from the momentum equation $(7 b)$. This leads to the useful result

$$
\frac{1}{p} \frac{d q}{d x}=\left(M^{2}-1+\tau / p\right) \frac{d u}{d x}
$$

However, it should be remembered that (18) is only an approximate relation since (6) was used in its derivation.

Using Eq. (18) together with the Chapman-Enskog value for $\rho<V_{x}^{3}$ given by (17) and substituting into (11), the "first" approximation for $\tau^{*}$ is found to be given by 


$$
\tau^{*}=\left[\frac{9}{5}\left(1-\frac{T}{p}\right)-\frac{7}{15} M^{2} \mu \frac{d u}{d x} .\right.
$$

Here again, we see that for $M_{s} \approx 1$ and $|\tau / p| \ll 1$ the limiting value of $\tau^{*}$ is $\frac{4}{3} \mu \frac{\mathrm{du}}{\mathrm{dx}}$. However, for this approximation the critical Mach number is given by

$$
M_{s}=\sqrt{27 / 7}=1.96
$$

which is an improvement over the previous value, but it is still not very exciting.

The simplest generalization to the Chapman-Enskog value is to assume $\mathrm{Q}$ is still a constant but that it takes on a different value $\alpha$, the value being determined by the vanishing of the critical Mach number. Following the same substitution as above, we now have

$$
\tau^{*}=\left[(1-\tau / p)+\frac{6 \alpha-5}{9-6 \alpha} M^{2}\right](3-2 \alpha) \mu \frac{d u}{d x} .
$$

For $\alpha=5 / 6$ the coefficient of the $\mathrm{M}^{2}$ term becomes zero and the expression no longer exhibits a critical Mach number. Our new approximation is then

$$
\mathrm{Q}=5 / 6,
$$

and we obtain a new law relating stress and deformation rate given by

$$
\tau^{*}=(1-\tau / p) \frac{4}{3} \mu \frac{d u}{d x}
$$

A more symmetric form of the equation is given by

$$
\tau^{0}=\tau^{*} /(1-\tau / p) \text {, }
$$

where $\tau^{0}$ is the Navier-Stokes value (8a).

For Maxwell molecules $\tau^{*}=\tau$ and Eq. (21) together with (7c) and (7e) yield the extremely simple constitutive relations 


$$
\begin{aligned}
& \tau^{o}=\tau /(1-\tau / p) \\
& q^{o}=q /(1-\tau / p),
\end{aligned}
$$

where in the second equation we have assumed $\mu \mathrm{c}_{\mathrm{p}} / \mathrm{k}=3 / 4$, consistent with approximation (6), in order that the Navier-Stokes relations are fully recovered for small values of $|\tau / p|$.

A plot of Eq. (22a) is presented in Fig. 1, where the dimensionless strain rate $\tau^{0} / p$ is plotted against the dimensionless stress $\tau / p$. Since $\tau / p$ is always a negative number in a shock wave, only one quadrant of the coordinate system is shown in the figure. The upper limit on the value of $|\tau / p|$. is well defined as discussed by Liepmann, Narasimha and Chahine ${ }^{4}$; i. e., as a result of the definition of $\tau / p$ kinetic theory limits its value to -2 , and manipulation of the momentum equation shows that its value is limited to $-3 / 2$ which occurs for an infinitely strong shock wave. The figure therefore shows that the values of $-\tau^{\circ} / \mathrm{p}$ predicted by Eq. (22a) are always less than the Navier-Stokes values and the ratio to the Navier-Stokes value reaches a minimum of $2 / 5$. Consequently, shock wave thickness as predicted by (22a) should be greater than that predicted by the Navier-Stokes relations.

Although the constitutive relations (22a) and (22b) are remarkably simple, which suggests a certain validity in itself, it is appropriate to question the approximation (20) in view of the disagreement with the Chapman-Enskog value, $\mathrm{Q}=3 / 5$. However, since the Chapman-Enskog value is in effect the result of a special limit proeess, it's quite conceivable that other values could be obtained for other conditions. 
For very large shock Mach numbers, the flow at the upstream end of a . shock wave where significant molecular collisions first begin to occur is akin to a molecular beam, because of the developing difference between the parallel and perpendicular components of the thermal motion of the molecules. If we consider an actual molecular beam as an example, for which $\left\langle\mathrm{V}_{\mathrm{x}}^{2}\right\rangle \neq 0$ and $\left\langle\mathrm{V}_{\mathrm{y}}^{2}\right\rangle=0$, then definitions (13a), (13b) and (14) show that $\mathrm{Q}$ takes on its maximum value for this case and its value is given by

$$
Q=q^{\prime \prime} /\left(q^{\prime \prime}+q^{L}\right)=1
$$

where $q^{+}=0$. Likewise the definition shows that the absolute minimum value of $Q$ would be zero. Therefore one would expect $Q$ to assume values between these two extremes in an actual flow field.

In fact, it would even be difficult to imagine that $Q$ could be less than $1 / 3$ in a shock wave, since this would mean that the transport of energy associated with each transverse degree of freedom exceeds the transport of energy associated with the parallel degree of freedom. If the value $1 / 3$ is truly approached somewhere in a shock wave, it seems reasonable to expect to find it at the subsonic end of a strong shock wave where the Mach number is low and the distribution function loses its preferential direction.

Likewise, the upper limit $Q=1$ is probably not attained in a shock wave since the upstream portion is never precisely a molecular beam regardless of how large $M_{s}$ becomes; and it is quite possible that the requirement that the critical Mach number vanish in Eq. (11), which led to the result $Q=5 / 6$, in fact selects the maximum permissible value of $Q$ in a shock wave.

If we now assume that $Q$ lies in the range 


$$
1 / 3 \leq \mathrm{Q} \leq 5 / 6
$$

with large values on the supersonic side and small values on the subsonic side of a shock wave, it becomes clear that the Chapman-Enskog value $Q=3 / 5$ could very likely be the proper value for the portion of the flow where the local Mach number is unity.

The next level of approximation to (15) is therefore to establish a functional form for $Q$ beyond merely a constant. To do this one must identify the important independent variables entering the expression for Q. We have already suggested that the Mach number is important is arriving at (25). The constitutive relations show that $T / p$ is a very important quantity in a shock wave, so it is likely that it too is an important variable in expressing $Q$. If we chose to add to this list the second Chapman-Enskog parameter $\mathrm{q} / \mathrm{pa}$, we find however that it is not a new variable in a shock wave, since it is a combination of $M$ and $\tau / p$, i. e.,

$$
q / p a=T u / p a=(T / p) M
$$

Since Eq. (26) lists the most important variables in a shock wave, it is reasonable to assume that a suitable approximation would consist of the following expression

$$
Q=Q(M, T / p)
$$

with (25) setting the upper and lower limits on $Q$ for all $M$ and $T / p$.

Several important details concerning the functional form of $Q$ could now be established by substituting (15) into (11), using the assumed form (27), and studying the various limits of the resulting expression. With this information, a particular algebraic expression could be chosen for (27) (this would be equivalent to choosing $\mathrm{f}$ ); and thus an improved approximation for $\tau^{*}$ would then be obtained. 
The important feature of this approximation would be that $T^{\circ} / p$ would depend on both $\tau / p$ and $M$ (or $q / p a)$ rather than merely on $\tau / p$ as given by (22a); this would be the first level of approximation at which the constitutive law for stress would also depend on the heat flux $q$, as one would expect in a nonequilibrium situation. Since the algebraic details for this case can become involved, it is appropriate to defer this discussion and first obtain a prediction of shock-wave profile based on the simpler constitutive relations (22a) and (22b). 


\section{SHOCK-WAVE STRUCTURE, $Q=5 / 6$}

The constitutive relations (22a) and (22b) were obtained using approximation (6), assuming Maxwell molecules, a Prandtl number of $3 / 4$, and the approximation $\mathrm{Q}=5 / 6$. The analysis to this point can be viewed as an effort to derive approximate constitutive relations for stress and heat flux for a gas passing through a shock wave, not to solve the full set of Eqs. (1). However, we can now return to the set given by (1) and replace Eqs. (1d) and (1e) with the approximate relations (22a) and (22b). In fact, since it is reasonable to assume that a material description of a gas cannot be very sensitive to the type of gas being considered (for example, the Navier-Stokes relations apply equally well to both monatomic and diatomic gases, as well to liquids), one can postulate that the above constitutive relations hold in general and solve the set for the appropriate viscosity law $\mu(T)$, ratio of specific heats $\gamma$, and Prandtl number $\operatorname{Pr}$ needed to describe the gas. Using this point of view, the appropriate set of equations to solve for shock structure is given by

$$
\begin{aligned}
& \rho u=m \\
& m\left(u-u_{1}\right)+p-p_{1}=\tau \\
& m\left(h+\frac{1}{2} u^{2}-H_{1}\right)=(\tau u-q) \\
& \tau^{o}=\tau /(1-\tau / p) \\
& q^{o}=q /(1-\tau / p) \\
& \tau^{o}=\frac{4}{3} \mu \frac{d u}{d x}
\end{aligned}
$$




$$
q^{0}=-k \frac{d T}{d x^{2}}
$$

to which one would add (4a) and (4b) for a thermally and calorically perfect gas. In the above set, the first three equations are integral forms of Eqs. (1a) to (1c), subscript 1 is used to indicate an upstream value, and $\mu$ and $k$ are assumed to be known functions of temperature.

As a result of the fact that the above expressions for $T$ and $q$ yield the following relation

$$
T^{0} / q^{0}=T / q
$$

we will find that one can use exactly the same method to solve the above set as that discussed by Gillarg and Paolucci ${ }^{6}$ for the solution of the Navier-Stokes equations. In fact, Eq. (29) guarantees the same behavior at the upstream and downstream singular points for the solution in the $T, u$ plane as that found by Gillarg and Paolucci for the Navier-Stokes equations.

Before proceeding with the analysis, it is quite useful to introduce an algebraic tracer $€$ as a coefficient before the $\tau / p$ term in both Eqs. (28d) and (28e) and then use the following scheme: If we set $\epsilon=0$, the above set of equations become identical to the Navier-Stokes equations; and if we set $\epsilon=1$, we recover the set of Eqg. (28).

Using the algebraic tracer $\epsilon$, we have from Eq. (28d)

$$
\tau^{0} / \mathrm{p}=(\tau / \mathrm{p}) /(1-\epsilon \tau / \mathrm{p})=(\tau u / p u) /(1-\epsilon \tau u / p u)
$$

Multiplying the momentum Eq. (28b) by $u_{,}$to obtain an expression for $\mathrm{Tu}$, and defining the dimensionless variables $\tilde{T}=T / T_{1}$ and $\tilde{u}=u / u_{1}$, the above equation 
can be written in the following form

$$
\tau^{o} / p_{1}=\tilde{T} \theta / \tilde{u}(\tilde{T}-\epsilon \theta)_{s}
$$

where

$$
\theta=\left[\tilde{\mathrm{T}}-\tilde{\mathrm{u}}\left(1+\gamma \mathrm{M}_{\mathrm{s}}^{2}(1-\tilde{\mathrm{u}})\right)\right]
$$

Simplification of the equation for the two cases $\epsilon=0$ and $\epsilon=1$ is obviously straightforward. Likewise, from Eq. (28d), we have

$$
q^{o} / p u=(q / p u) /(1-\epsilon \tau u / p u) .
$$

Using the energy Eq. (28c) to obtain $q$ and the momentum Eq. (28b) to obtain $\mathrm{Tu}$, the above equation can be written in the dimensionless form

$$
\mathrm{q}^{0} / \mathrm{p}_{1} \mathrm{u}_{1}=\tilde{\mathrm{T}}\left[\theta+\frac{\gamma}{\gamma-1}\left(1+\frac{\gamma-1}{2} \mathrm{M}_{\mathrm{s}}^{2}\left(1-\tilde{\mathrm{u}}^{2}\right)-\tilde{\mathrm{T}}\right)\right] /(\tilde{\mathrm{T}}-\epsilon \theta)
$$

If we divide Eq. (31) by Eq. (30) to obtain the differential equation for the solution curve in the $\tilde{T}, \tilde{u}$ plane, we see that the algebraic tracer $\epsilon$ cancels out, $i_{.}$e. , we obtain

$$
\frac{\mathrm{d} \tilde{\mathrm{T}}}{\mathrm{d} \tilde{\mathrm{u}}}=-\frac{4}{3} \operatorname{Pr}(\gamma-1) \mathrm{M}_{\mathrm{s}}^{2}\left[\theta+\frac{\gamma}{\gamma-1}\left(1+\frac{\gamma-1}{2} \mathrm{M}_{\mathrm{s}}^{2}\left(1-\tilde{\mathrm{u}}^{2}\right)\right)-\tilde{\mathrm{T}}\right] \tilde{\mathrm{u}} / \theta
$$

Therefore the integral curve in the $\tilde{T}, \tilde{u}$ plane is exactly the same as that for the Navier-Stokes solution. However, the velocity gradient and the temperature gradient are quite different for the two solutions as shown by Eqs. (30) and (31) for the two cases $\epsilon=0$ and $\epsilon=1$.

Once Eq. (32) has been integrated to obtain the universal curve $\tilde{T}=\tilde{T}(\tilde{u})$ for a particular combination of $\mathrm{M}_{\mathrm{s}}, \gamma$ and $\operatorname{Pr}_{3} \mathrm{Eq} .(30)$ can be evaluated to 
obtain the dimensionless velocity gradient $\tau^{0} / \mathrm{p}_{1} \mathrm{M}_{\mathrm{s}}$, for either the present solution $(\epsilon=1)$ or the Navier-Stokes solution $(\epsilon=0)$. Figure 2 presents a comparison of the two solutions for several different shock Mach numbers and for the case $\gamma=5 / 3, \operatorname{Pr}=2 / 3$. The figure shows that the two solutions are nearly identical for $M_{\mathbf{s}}=1.5$. However, they are quite different for the stronger shock waves, with the present solution having a significantly smaller value for the maximum velocity gradient. It can be seen from the figure that most of the change occurs on the supersonic side of the shock wave $(\tilde{u} \approx 1)$ with very little change occurring on the subsonic side.

In order to generate a shock wave profile from the data in Fig. 2, it is necessary to specify the temperature dependence of the coefficient of viscosity $\mu$. Here we assume a power law dependence for the viscosity, $\mu \sim T^{\omega}$, with $\omega=0.75$ (to represent argon as discussed below). Figures 3 and 4 give a comparison of several density profiles for the two solutions. The spatial variable $\mathrm{x}$ has been nondimensionalized by dividing by the upstream mean-free path length $\lambda_{1}=\frac{16}{5} \sqrt{\frac{\gamma}{2 \pi}} \frac{\mu_{1}}{\rho_{1} a_{1}}$. The two profiles were arbitrarily matched at the subsonic side because of the suggestive appearance of the curves in Fig. 2.

The figures show that the two solutions yield nearly identical results for $\mathrm{M}_{\mathrm{S}}=1.5$ and the difference in the two profiles becomes greater as the shock Mach number increases. The increase in shock-wave thickness over the NavierStokes value shown by the present solution is definitely in the proper direction for agreement with experimental results. However, we also see from Fig. 4 that considerable asymmetry develops in the predicted density profile for the 
larger values of $M_{S}$; and this is not in agreement with experimental results. If a detailed comparison is made with experimental density profiles, one finds that the present solution lies too close to the Navier-Stokes solution at the subsonic end of the shock wave, i.e., we must conclude that the constitutive relations require further corrections for $|\tau / p|$ small. 


\section{EXTENSION, $Q=Q(M)$}

The first step beyond the approximation $Q=5 / 6$ must include the most important functional dependence in the representation for $Q$. The discussion leading to Eq. (27) suggests that $M$ and $T / p$ are important variables in representing $Q$, but no judgment was made as to which variable is the most important. By assuming initially that both must be included, we can arrive at an equation, Eq. (36), which will show that the functional dependence on $M$ is far more essential than the dependence on $\tau / p$.

Assuming the representation $Q=Q(M, T / p)$, the $x$-derivative of the product $\mathrm{qQ}$ is given by

$$
\frac{d}{d x} q Q=Q \frac{d q}{d x}+q\left[Q \frac{d M}{M x}+Q / p \frac{d}{d x}(T / p)\right]
$$

where $Q_{M}$ and $Q_{T / p}$ are partial derivatives with respect to $M$ 'and $\tau / p$ respectively. Using the definitions for $M$ and the isentropic speed of sound $a$, we have

$$
\frac{d M}{d x}=\frac{d}{d x}\left(\frac{u}{a}\right)=\frac{1}{a} \frac{d u}{d x}-\frac{u}{a} \frac{d}{d x} \sqrt{\frac{\gamma p}{\rho}}
$$

which by virtue of the continuity Eq. (28a) allows one to write

$$
\frac{d M}{d x}=\frac{1}{a}\left[\frac{d u}{d x}-\frac{1}{2 p} \frac{d}{d x}(p u)\right]
$$

Multiplying the momentum Eq. (28b) by $u$ and using approximation (6) together with Eq. (18), the above expression becomes 


$$
\frac{d M}{d x}=\frac{1}{a}\left(\frac{1}{3} M^{2}+1\right) \frac{d u}{d x}
$$

Similarly for the $\mathrm{x}$-derivative of $\tau / \mathrm{p}$, we can write

$$
\frac{d}{d x} T / p=\frac{d}{d x}(q / p u)=\frac{1}{p u}\left[\frac{d q}{d x}-(\tau / p) \frac{d}{d x}(p u)\right]
$$

Following the same procedure as above for determining the $\mathrm{x}$-derivative of $\mathrm{pu}$, we have finally

$$
\frac{d}{d x} T / p=\frac{1}{u}\left[\left(M^{2}-1\right)+\left(\frac{2}{3} M^{2}+1\right) \tau / p\right] \frac{d u}{d x}
$$

Using the derivatives given by (34) and (35) together with the expression derived in (18) for $\frac{\mathrm{dq}}{\mathrm{dx}}, \mathrm{Eq}$. (33) can be written as

$$
\begin{aligned}
& \frac{1}{\mathrm{p}} \frac{\mathrm{d}}{\mathrm{dx}} \mathrm{qQ}=\left\{\mathrm{Q}\left(\mathrm{M}^{2}-1+\tau / \mathrm{p}\right)+\mathrm{Q}_{\mathrm{M}}\left(\frac{1}{3} \mathrm{M}^{2}+1\right) \mathrm{M} \tau / \mathrm{p}+\mathrm{Q}_{\tau / \mathrm{p}}\left[\left(\mathrm{M}^{2}-1\right)\right.\right. \\
& \left.\left.\quad+\left(\frac{2}{3} \mathrm{M}^{2}+1\right) \tau / \mathrm{p}\right\}\right\} \frac{\mathrm{du}}{\mathrm{dx}},
\end{aligned}
$$

where the approximation $q=\tau u$ has again been used. With the aid of definition (15) and the above equation, the expression for $\tau^{*}$ given by Eq. (11) yields the result

$$
\begin{aligned}
& 4 \tau^{*} / T^{0}=\left[(9-6 Q)-(5-6 Q) M^{2}\right]-\left[(9-6 Q)-2 Q_{M}\left(M^{2}+3\right) M\right. \\
& \left.-2 Q_{T / p}\left(M^{2}-1\right)\right] \tau / p+\left[2 Q_{T / p}\left(2 M^{2}+3\right)\right](\tau / p)
\end{aligned}
$$

Since $|\tau / p| \leq 3 / 2$ for all shock Mach numbers and since in general $\tau^{*} \approx \tau$, therefore $\tau^{*} / \tau^{0}$ must be bounded; and this fact allows several important conclusions to be drawn concerning the functional form of Q. If it were assumed 
that $Q$ is a function of $T / p$ alone, then the last two bracketed terms in Eq. (36) would increase with $\mathrm{M}^{2}$ and $\tau^{*} / \tau^{\circ}$ would not be bounded. Therefore $\mathrm{Q}$ cannot be a function of $\tau / p$ alone. However, if it were assumed that $Q$ is a function of M alone, then it would only be necessary to require $Q_{M}$ to vary like $M^{-3}$ for large $M$ and $\tau^{*} / \tau^{\circ}$ would be bounded. Therefore the dependence on $M$ is necessary in the functional representation for $Q$, as long as $Q$ is not a constant. Proceeding on the basis that $Q=Q(M)$ is the appropriate generalization beyond the previous approximation $Q=5 / 6$, Eq. (36) simplifies to read

$$
4 \tau^{*} / T^{0}=\left[(9-6 Q)-(5-6 Q) M^{2}\right]-\left[(9-6 Q)-2 Q_{M}\left(M^{2}+3\right) M\right] \tau / p
$$

Returning to the requirement that $\tau^{*} / \tau^{0}$ be bounded, one concludes from (37) that $Q(M)$ must exhibit the following behavior for large $M$

$$
\begin{aligned}
& \lim _{M \rightarrow \infty} M^{2}[5-6 Q]=c_{1} \\
& \lim _{M \rightarrow \infty} M^{3} Q_{M}=c_{2},
\end{aligned}
$$

where $c_{1}$ and $c_{2}$ are constants. If we further assume $c_{1}$ and $c_{2}$ are nonzero, the functional form of $Q(M)$ for large $M$ can be established in greater detail. In particular, Eq. (38a) shows that the difference $[5 / 6-Q]$ must vary like $\mathrm{M}^{-2}$. If in addition we agree that $Q(1)$ must be the Chapman-Enskog value $3 / 5$ and that $Q(M)$ is a monotonic function, we can write

$$
Q(M)=15 M^{2} /\left(7+18 M^{2}\right)
$$

as a reasonable approximation for $Q(M)$ satisfying the cited requirements.

It is obvious that Eq. (39) satisfies Eqs. (38a) and (38b) since 


$$
[5 / 6-Q]=35 / 6\left(7+18 M^{2}\right)
$$

and therefore $Q_{M} \sim M^{-3}$ which is required by $(38 b)$. In arriving at Eq. (39) it was assumed that the simplest algebraic expression satisfying the above conditions would be adequate.

Figure 5 presents a plot of the function $Q(M)$ defined by Eq. (39). The curve was not drawn for $M<1 / 2$ since the minimum Mach number behind a shock wave in a monatomic gas is approximately $1 / 2$. Although the proposed function has the correct behavior for large $M$ (in the sense that $c_{1}$ and $c_{2}$ are nonzero), it is not obvious that it has the correct behavior near $M=1$, which unfortunately cannot be determined with the information at hand. Of particular concern is the large variation exhibited by the proposed function about the Chapman-Enskog value, which possibly is a result of the simple algebraic expression chosen.

The validity of the approximation could be checked quite conclusively by including in Fig. 5 data obtained from numerical solutions of the Boltzmann equation or any suitable approximation thereto. The numerical data, however, would have to be obtained for a wide range of shock Mach numbers and from solutions for which the shock wave is accurately described in the wings of the profile (where the Mach number reaches either bounding value) in order to adequately check the proposed function. Numerical data in a preliminary form was very kindly made available to the authors recently by S. M. Yen who together with B. L. Hicks has used the Monte Carlo method to evaluate the Boltzmann collision integral for hard sphere collisions. The general trend of this preliminary data 
has provided considerable encouragement for the use of Eq. (39), and in particular it has helped to dispel certain misgivings which were originally held about the large variation in the function near $M=1$ from the Chapman-Enskog value. (Because of the preliminary nature of the data, it was not felt that it would be appropriate to include these data in Fig. 5.)

Turning our attention back to Eq. (37) and using Eq. (39), we again find a relatively simple constitutive relation for stress, i. e.,

$$
\tau^{0}=\tau * /(F-G \tau / p),
$$

where $F$ and $G$ are functions of $M$ given by

$$
\begin{aligned}
& F=\left(63+37 M^{2}\right) / 4\left(7+18 M^{2}\right) \\
& G=3\left(147+126 M^{2}+292 M^{4}\right) / 4\left(7+18 M^{2}\right)^{2} .
\end{aligned}
$$

Constitutive relations for $\tau$ and $q$ can again be obtained by assuming the gas is composed of Maxwell molecules and by following the same algebraic manipulation leading to Eqs. (22a) and (22b). It is of interest, and of importance to the development below, that the only change that occurs in Eqs. (22) is the quantity $(1-\tau / p)$ is replaced by $(F-G \tau / p)$. Since $F$ and $G$ are bounded by the values $1.57 \geq F \geq 0.357$ $1.42 \geq G \geq 0.676$

for the Mach number range $0.5 \leq M \leq \infty$, one can see the modification is not one of a fundamental nature.

The equation relating stress and deformation rate for Maxwell molecules, obtained from Eq. (40), is plotted in Fig. 6 for fixed values of M. Here we see the Navier-Stokes relation is recovered only for the case $M=1$ and $|T / p|$ 
small. The fact that the Navier-Stokes relation is not recovered far upstream and far downstream from the shock wave where $|\tau / p|$ is small and $M \neq 1$ comes as a surprise from both a physical and a mathematical point of view. This difference could, in part, be attributed to the approximation used for $Q$ and the fact that the $\tau / p$ dependence was ignored. However, the difference appears in precisely the correct direction to remove the asymmetry from the shock-wave profile that was noticed in Figs. 3 and 4 with the simpler theory. This fact in itself is a strong argument in favor of employing the constitutive relation for stress as displayed in Fig. 6 and checking the accuracy with which the new theory can predict shock-wave profile.

Since Eq. (29) still holds and Eq. (40) introduces $M$ as the only new variable, the same method can be used to compute shock-wave structure with the new approximation as was discussed in the above section for the simpler theory; Eq. (32) is of course exactly the same and Eqs. (30) and (31) must be modified in a trivial manner to include the functions $F$ and $G$ appearing in Eq. (40). Figures 7 and 8 present the results of several such calculations for the density profile through a shock wave for the case $\gamma=5 / 3, \operatorname{Pr}=2 / 3$, and $\omega=0.75$. A suitable value of $\omega$ for low temperature argon is 0.816 which has been used by a number of investigators since the work of Gillarg and Paolucci. If shock-wave profiles are to be computed for $M_{S} \leq 10$, then a value is need for a much greater temperature range. For the temperatures $\mathrm{T} \leq 10,000^{\circ} \mathrm{K}$, a suitable "average" value appears to be $\omega=0.75$, according to the calculations for argon by Amdur and Mason ${ }^{7}$ and the experimental data obtained by Camac 
and Feinberg ${ }^{8}$, Collins and Mennard ${ }^{9}$, and Willeke ${ }^{10}$. The dashed curves appearing in the two figures are plots of experimental data for argon obtained by B. Schmidt ${ }^{11}$ using an electron beam densitometer and the 17-inchdiameter shock tube at Caltech. The theoretical and experimental density profiles were arbitrarily matched at the point $\left(\rho-\rho_{1}\right) /\left(\rho_{2}-\rho_{1}\right)=0.5$.

Two observations become immediately evident on comparing the theoretical and experimental curves in the figures. First, the theory predicts the experimental data rather well over the entire Mach number range from 2.8 to 8 ; and second, the theoretical profiles no longer exhibit the asymmetry that was present in Figs. 3 and 4. A close inspection of the figures shows that the theoretical profiles are slightly thinner than the experimental profiles in each case and the fit is slightly better for the larger Mach numbers. The value of $\omega$ used in the calculations could certainly be adjusted to improve the fit still further, especially for the lower Mach numbers, but in view of the uncertainty associated with the proper value of $\omega$ for a wide temperature range in argon and in view of the further improvements that could be incorporated in the present theory, it seems advisable to judge the theory on the basis of the present comparison alone. On this basis it is quite clear that the constitutive relation for stress exhibited in Fig. 6 is more nearly correct than that displayed in Fig. 1, and therefore any further improvement in the theory would probably yield results in closer agreement with Fig. 6 . This again raises the very interesting question of why the Navier-Stokes relations are not recovered far ahead of the shock wave and far behind the shock wave where the flow is nearly in equilibrium, since most physical arguments would tend to favor the opposite conclusion. 


\section{DISCUSSION}

In view of the favorable comparisons exhibited by Figs. 7 and 8 , further work to confirm or improve the present theory would be clearly justified on the grounds that it would contribute to the macroscopic interpretation of the physical processes taking place within a shock wave. For example, it would be extremely interesting, from the point of view of continuum mechanics, to check the constitutive relation for stress plotted in Fig. 6. This could be done with data obtained from numerical solutions of the Boltzmann equation or from calculations involving collisions between a finite number of particles, such as the work by Bird. ${ }^{12}$ The calculations would have to be carried out for several different values of $M_{S}$ since data corresponding to a single shock-wave profile would appear as a single loop in Fig. 6, starting from the origin and ending at the origin, with the curve for $M=1$ dividing the loop into two nearly-equal halves. Displayed in this form, the data should show a nonlinear dependence on $T / p$ and a Mach-number dependence about the locus of points for $M=1$. If Fig. 6 is approximately correct, the trajectory of each loop near the origin, for large values of $\mathrm{M}_{\mathrm{s}}$, would have a larger slope for the supersonic branch than for the subsonic branch. In terms of the present interpretation of $\mu(T)$, this implies that the Navier-Stokes relation for stress does not apply at the two ends of a shock wave.

A possible resolution of the problem could come from a more careful treatment of Eq. (40), e.g. , for $|\tau / p| \ll 1$ we have 


$$
\tau^{*}=\mathrm{F}^{0}{ }^{0}=\frac{4}{3} \mathrm{~F} \mu(\mathrm{T}) \frac{\mathrm{du}}{\mathrm{dx}}
$$

The procedure has been to assume Maxwell molecules (i. e., $T^{*}=\tau$ ) and then to assume $\mu(T)$ does not have to be restricted to the temperature dependence $T$ characterizing Maxwell molecules; obviously the two sides of Eq. (41) are not treated equally in such a procedure. An important extension to the present work would therefore be to relate $\tau^{*}$ to $\tau$ (and possibly $q$ ) for a general inversepower intermolecular potential so that compatible expressions for $\tau^{*}$ and $\mu(T)$ could be used; and thus physical interpretation of (41) would become more meaningful.

Figure 5 would likewise provide a physically interesting area for comparison with numerical solutions. Namely, the quantity $Q$ expresses the ratio between 1) the rate of flux of thermal energy contained in the degree of freedom which is parallel to the direction of propagation of a shock wave and 2) the total rate of flux of thermal energy. This information is of course useful in constructing a picture of how the distribution function changes from an upstream to a downstream position. In the case of Fig. 5., it would be desirable to determine whether the representation $Q=Q(M, \tau / p)$ would be necessary or whether the present approximation $Q=Q(M)$ is sufficient.

A final check on the present theory would be to review the approximation $q=T u$, or equivalently $H=$ constant, used in deriving the constitutive relations for stress and heat flux. The present approach is at least self consistent in that the maximum variation in the quantity $\left(\mathrm{H}-\mathrm{H}_{1}\right) / \mathrm{H}_{1}$ for all of the profiles shown 
in Figs. 7 and 8 is about $2 \%$. However, this is not surprising since the integral curve $\tilde{T}=\tilde{T}(\tilde{u})$ is the same as that for the Navier-Stokes solution, and therefore the relation $H=H(\tilde{u})$ should also be the same.

The constitutive relation for stress has been discussed in terms of the independent variables $\tau / p$ and $M$ rather than the equivalent combination of variables $\tau / p$ and $q / p a$ (see Eq. (26)), because of the fact that one can recover the case $M=1$ so much more easily. In terms of a physical description of a gas, it would seem that $\mathrm{T} / \mathrm{p}$ and $\mathrm{q} / \mathrm{pa}$ would be a more meaningful combination, since the velocity of the fluid does not appear in either variable. This raises the interesting possibility that the constitutive relations written in this form could also be applied to nonsteady, one-dimensional flows, e.g., to study the reflection of a shock wave from a wall, to analyze the nonsteady development of a shock front, or to study the attenuation of ultrasonic waves in a gas. 


\section{REFERENCES}

1. H. M. Mott-Smith, Phys. Rev. 82,885 (1951).

2. L. Lees, J. Soc. Indust. Appl. Math. 13, 278 (1965).

3. W. G. Vincenti and C. H. Kruger, Jr., Introduction to Physical Gas Dynamics (John Wiley and Sons, Inc., New York, 1965), p. 364.

4. H. W. Liepmann, R. Narasimha, and M. Chahine, Phys. Fluids $\underline{5}, 1313$ (1962).

5. F. S. Sherman and L. Talbot, in Rarefied Gas Dynamics, edited by F. M. Devienne (Pergamon Press, New York, 1960).

6. D. Gilbarg and D. Paolucci, J. Ratl. Mech. Anal. 2, 617 (1953).

7. I. Amdur and E. A. Mason, Phys. Fluids 1,370 (1958).

8. M. Camac and R. M. Feinberg, Avco-Everett Research Report 168 (1963).

9. D. J. Collins and W. A. Mennard, Jour, of Heat Trans, 88, 52 (1966).

10. K. Willeke, Ph. D. thesis, Stanford University (1969).

11. B. Schmidt, J. Fluid Mech. (to be published).

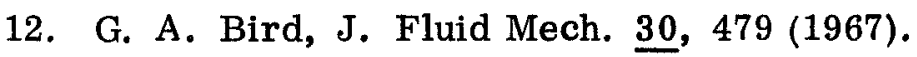




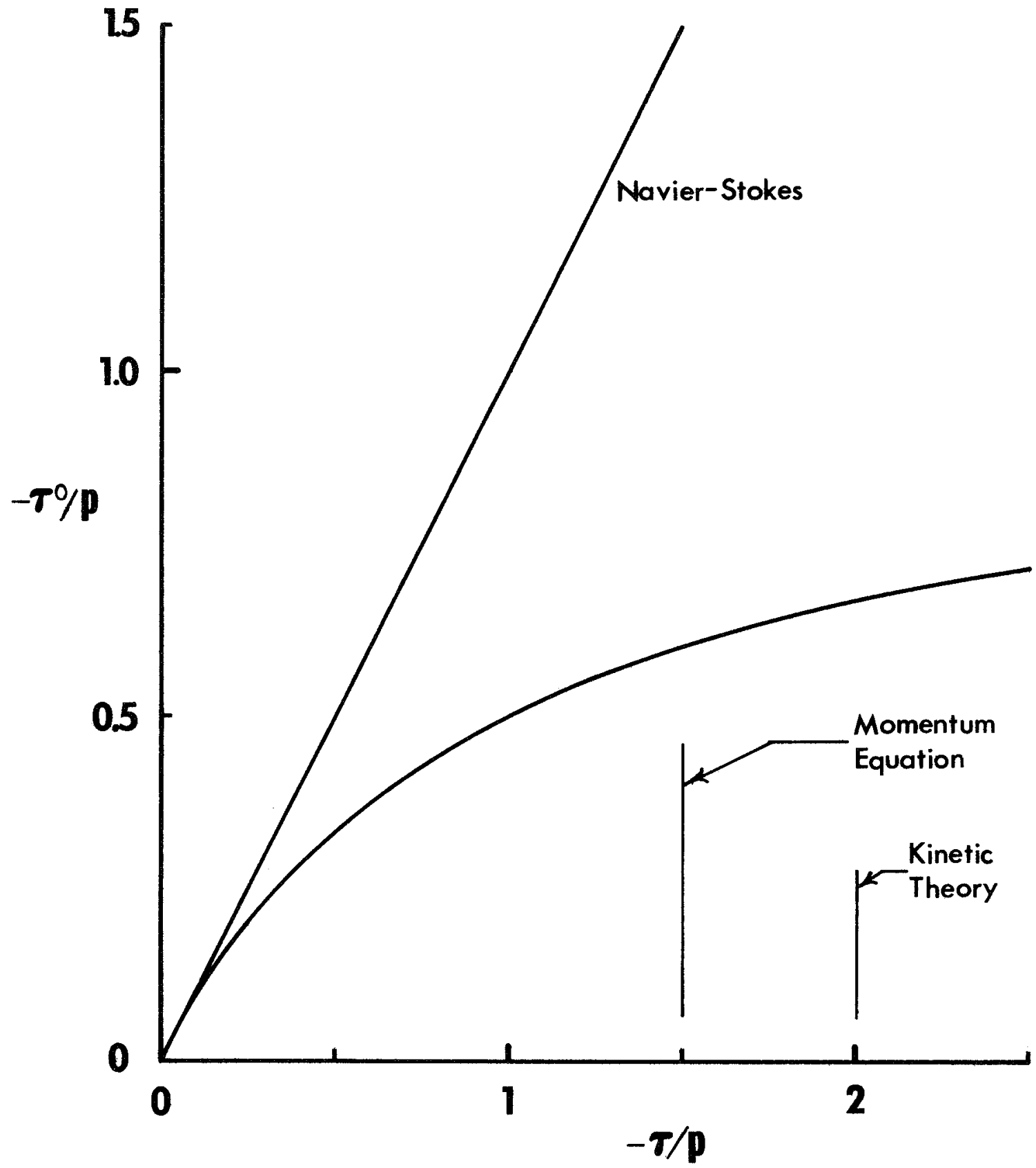

Fig. 1. Relation between nondimensional stress $\tau / p$ and nondimensional strain-rate $\tau^{\circ} / \mathrm{p}$ (for $Q=5 / 6$ ) compared with the Navier-Stokes relation. 


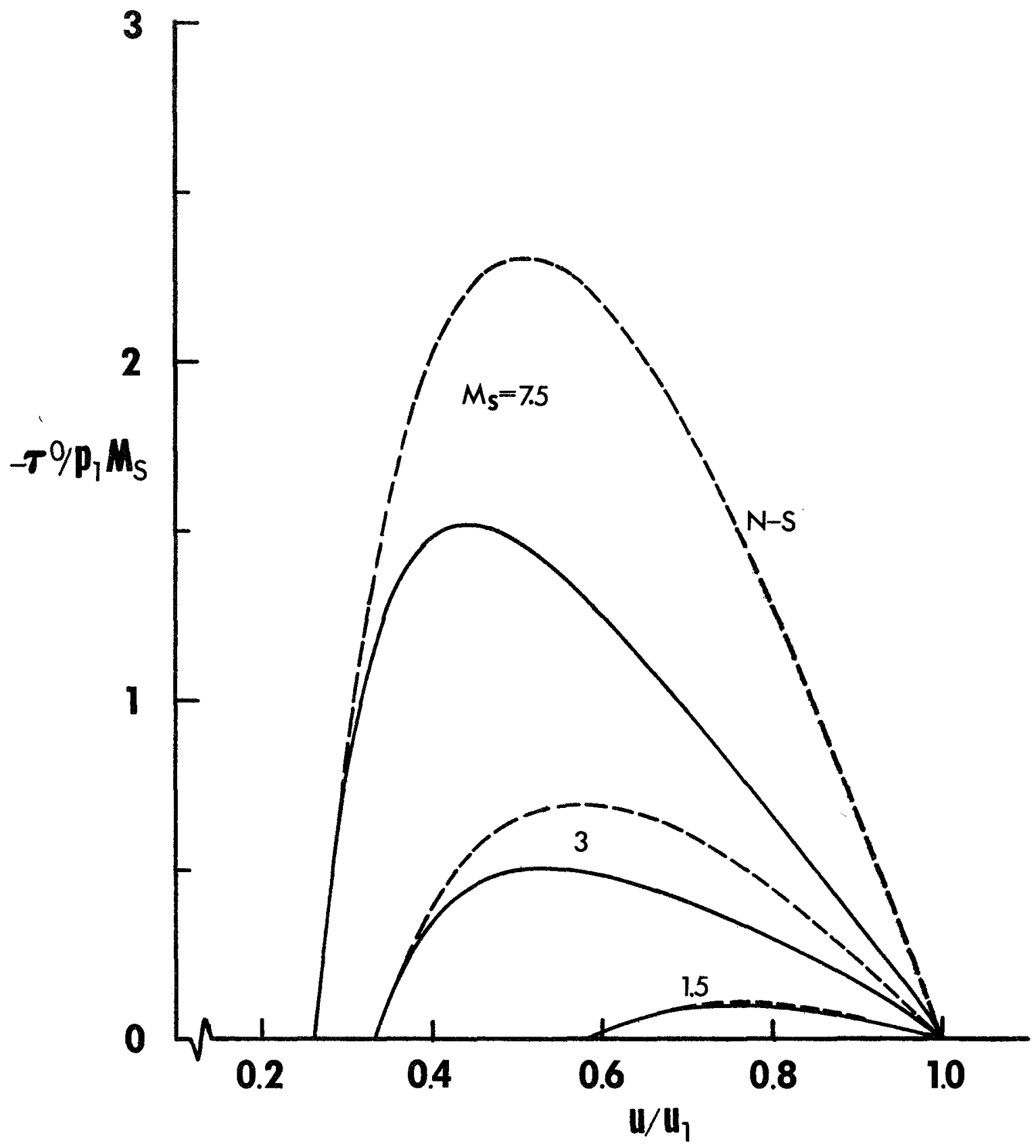

Fig. 2. Nondimensional velocity gradient for the Navier-Stokes solution and for the present solution (for $Q=5 / 6$ ). 

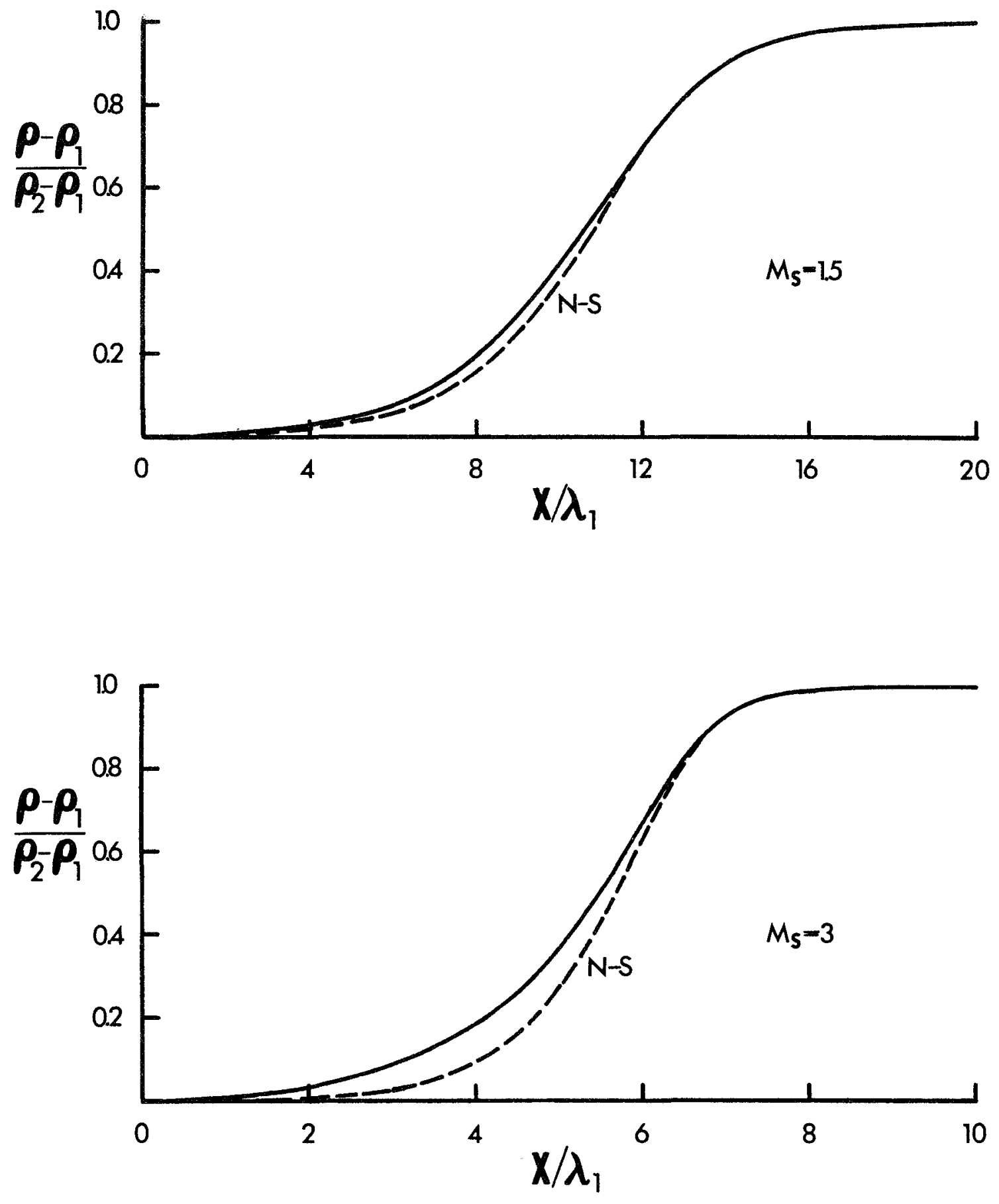

Fig. 3. Shock-wave density-profiles for $Q=5 / 6$ compared with the NavierStokes solution; $\operatorname{Pr}=2 / 3, \gamma=5 / 3$, and $\mu \sim \mathrm{T}^{3 / 4}$. 

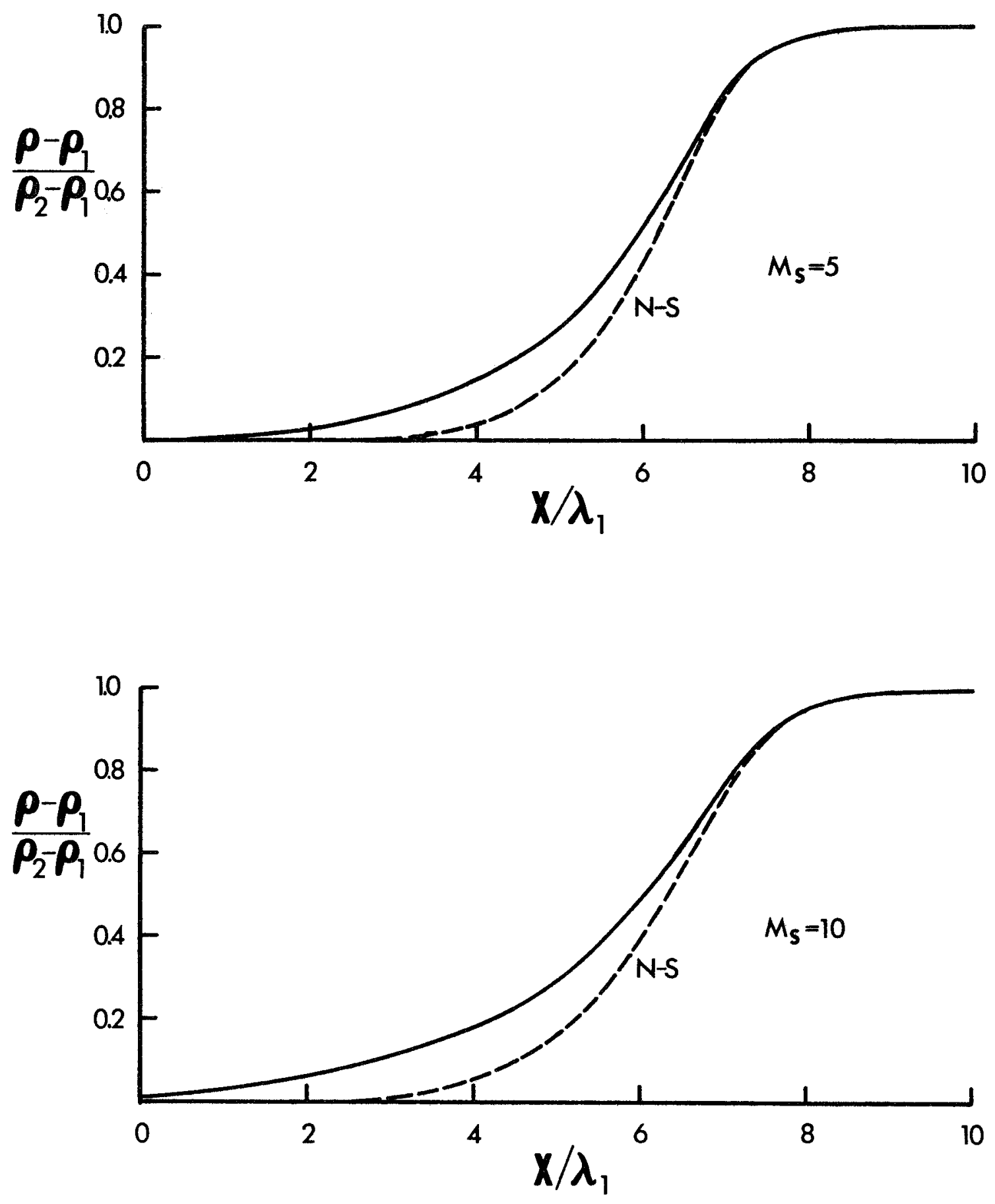

Fig. 4. Shock-wave density-profiles for $Q=5 / 6$ compared with the NavierStokes solution; $\operatorname{Pr}=2 / 3, \gamma=5 / 3$, and $\mu \sim \mathrm{T}^{3 / 4}$. 


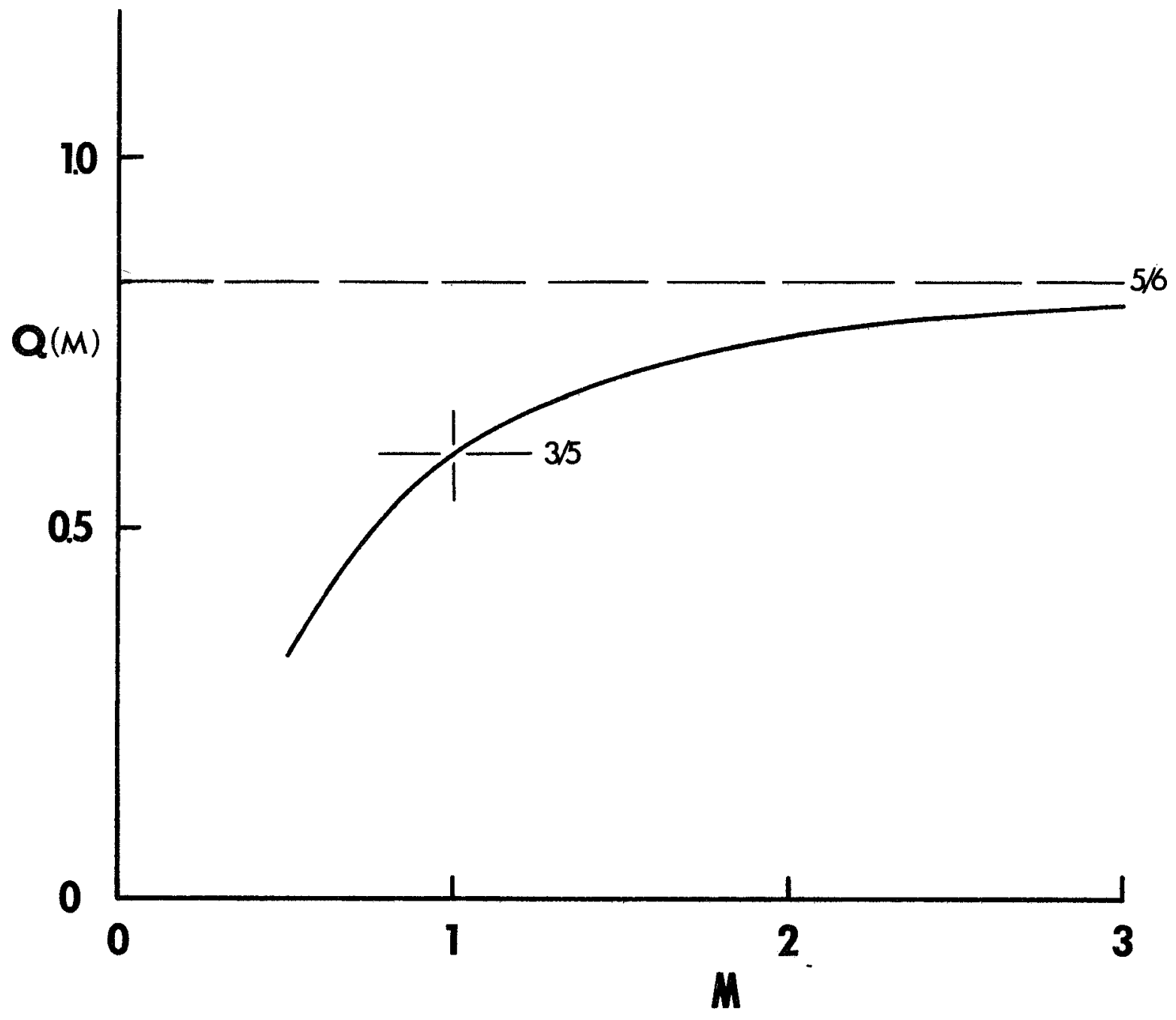

Fig. 5. Plot of the function $Q(M)$ defined by Eq. (39). 


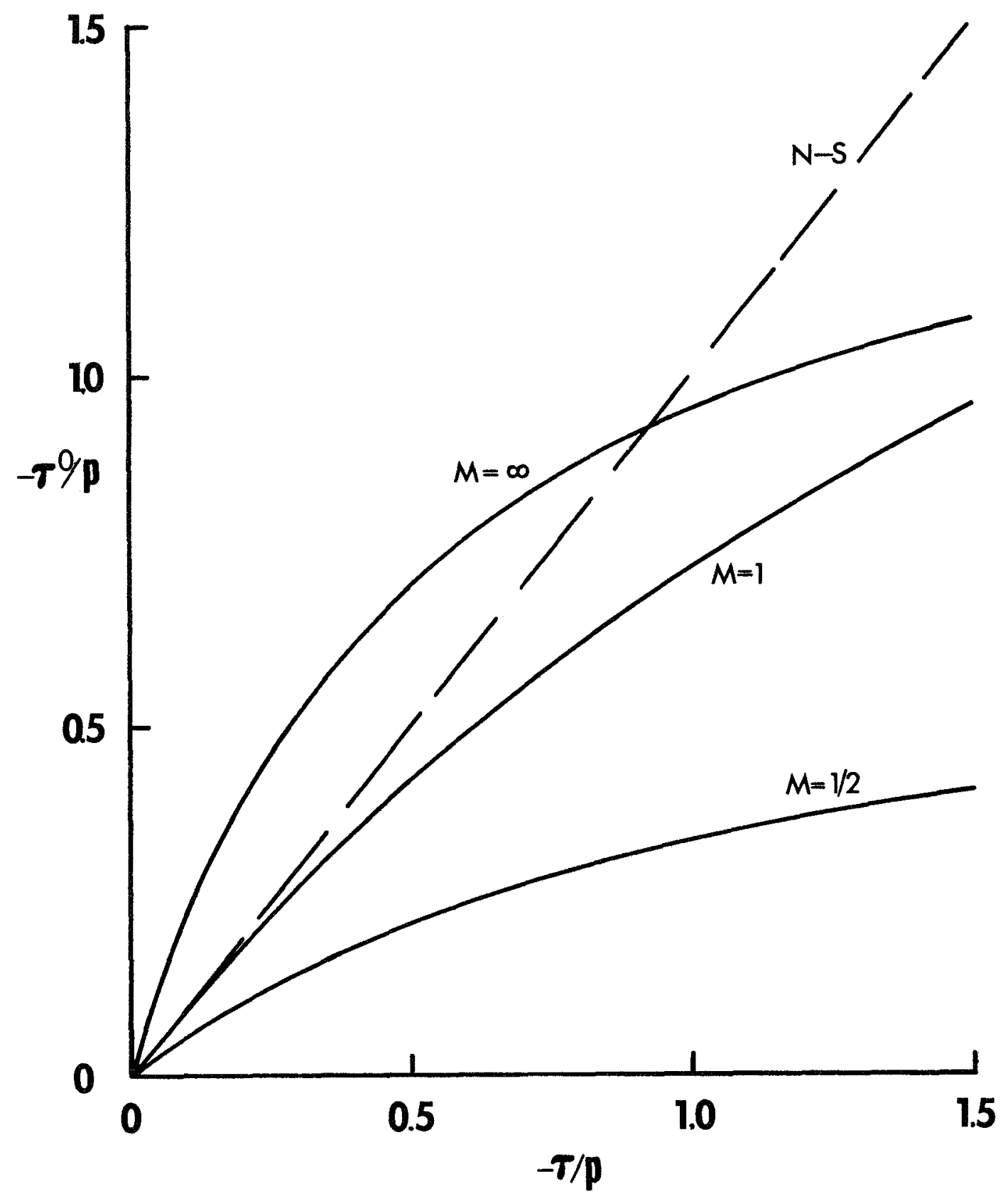

Fig. 6. Constitutive relation for stress based on the approximation $Q=Q(M)$, defined by Eq. (39). 

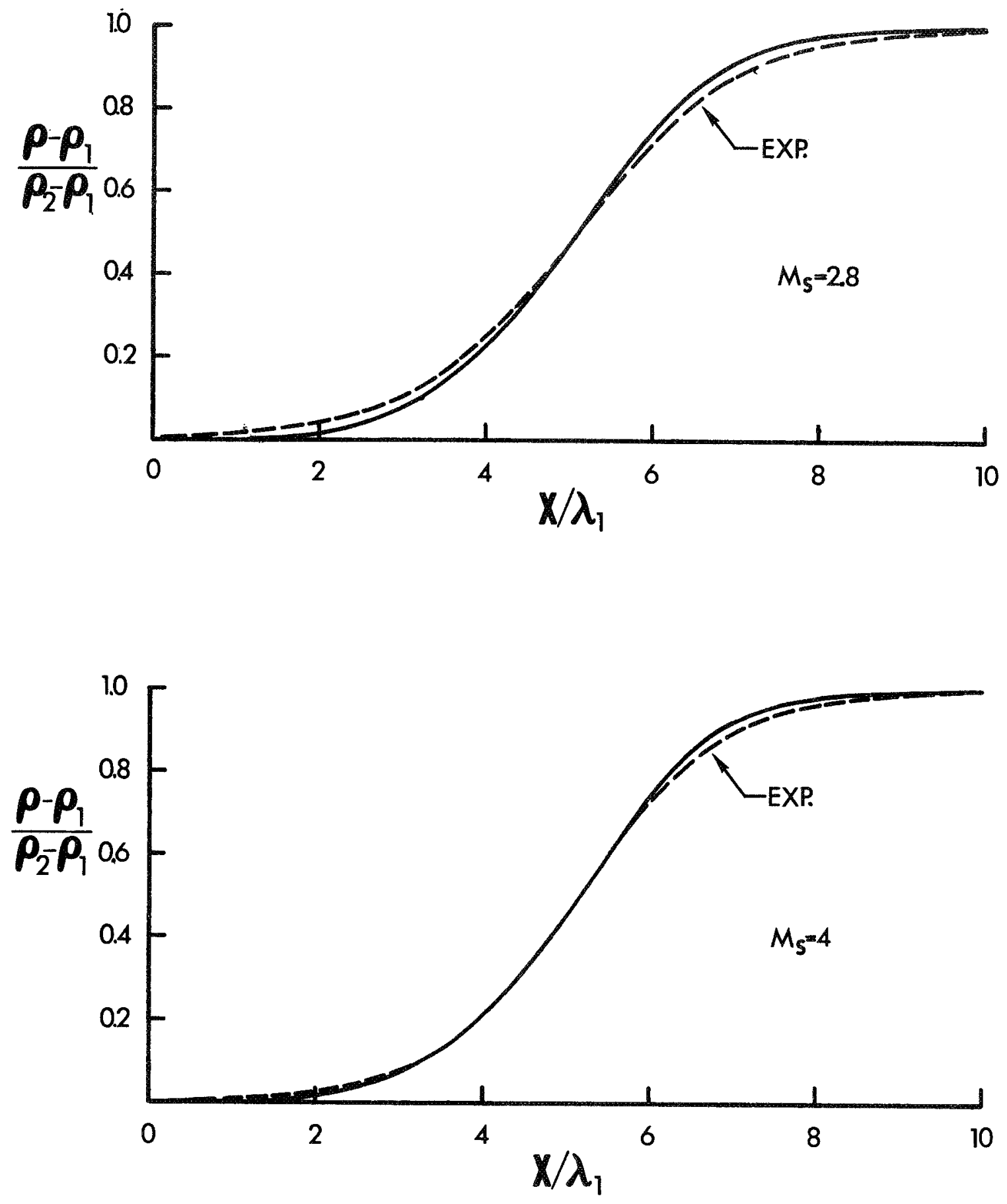

Fig. 7. Shock-wave density-profiles, for $Q=Q(M)$, compared with experimental data for argon obtained by Schmidt (Ref. 11); theory based on $\operatorname{Pr}=2 / 3$, $\gamma=5 / 3$, and $\mu \sim \mathrm{T}^{3 / 4}$. 

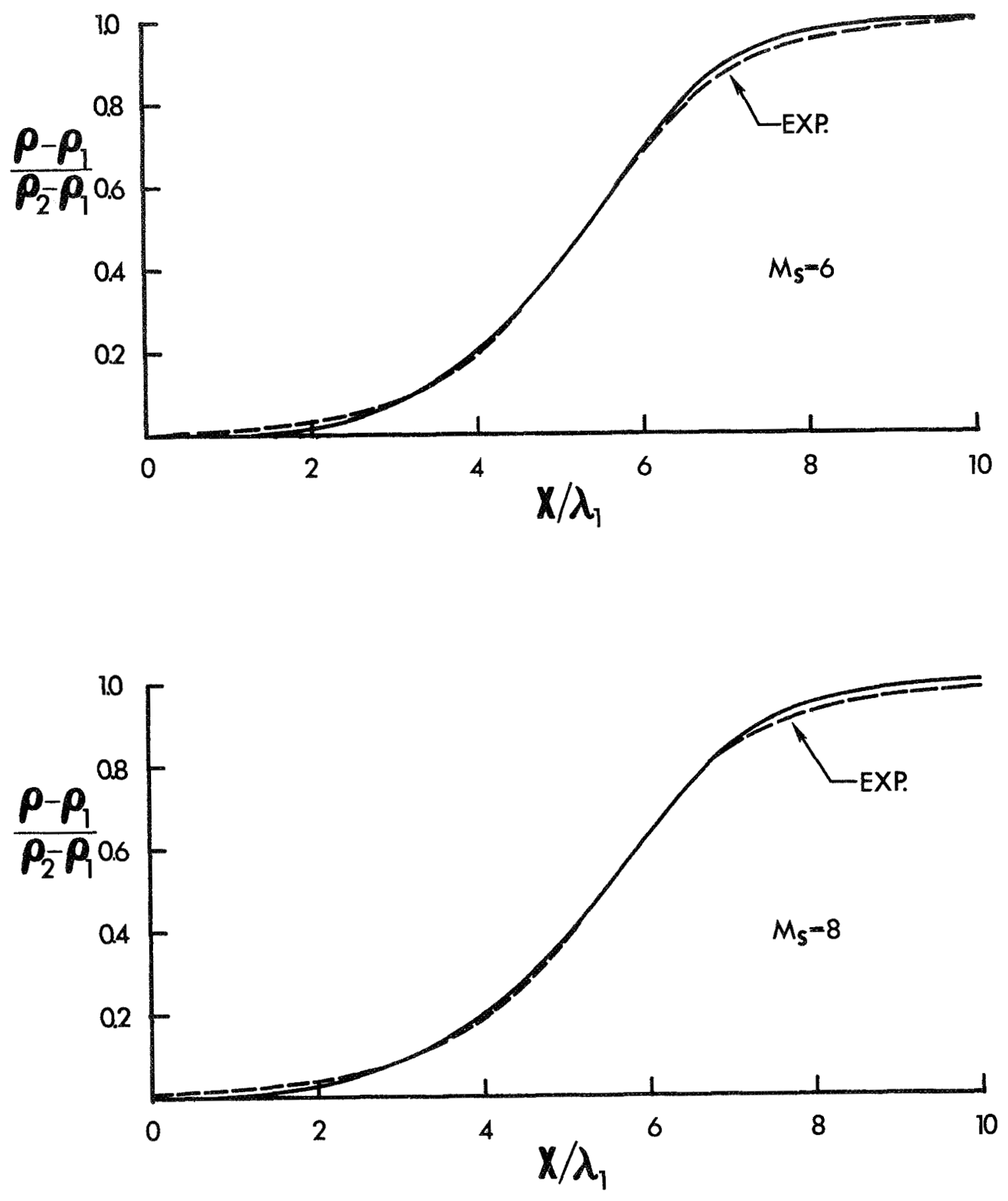

Fig. 8. Shock-wave density-profiles, for $Q=Q(M)$, compared with experimental data for argon obtained by Schmidt (Ref. 11); theory based on $\operatorname{Pr}=2 / 3$, $\gamma=5 / 3$, and $\mu \sim \mathrm{T}^{3 / 4}$. 